\title{
Crescimento e qualidade de mudas de faveleira em função do substrato e níveis de água ${ }^{1}$
}

\author{
Rosilvam Ramos de Sousa ${ }^{2}$, Antonio Lucineudo de Oliveira Freire ${ }^{3}$
}

\begin{abstract}
Resumo: Diante do cenário atual de degradação dos ecossistemas brasileiros, faz necessária a produção de mudas de qualidade visando à recuperação dos mesmos. No entanto, para atender a essa finalidade é necessário que se cause o mínimo de impacto ambiental possível. Nesse sentido, o uso de materiais alternativos na composição do substrato, bem como redução na quantidade de água na fase de viveiro são aspectos que devem ser considerados. Dessa forma, conduziu-se o presente trabalho com o objetivo de avaliar substratos e níveis de água no crescimento e na qualidade de mudas de faveleira, foram avaliados quatro misturas, sendo duas entre solo e esterco (2:1 e 3:1) e duas entre solo e rejeito de vermiculita (2:1 e 3:1) combinados com quatro níveis de água (100, 75, 50 e $25 \%$ da capacidade de vaso) no esquema fatorial 4 × 4 . Os tratamentos foram distribuídos em delineamento inteiramente cazualizado com quatro repetições por tratamento. Foram avaliados altura das plantas; diâmetro do coleto; as massas secas da parte aérea, das raízes, das folhas, do caule e total; taxa de crescimento absoluto; a razão altura/diâmetro e o Índice de Qualidade de Dickson. O substrato composto de solo + esterco bovino proporciona a obtenção de mudas de melhor qualidade. Recomenda-se o nível de água entre $75 \%$ e 100\% da capacidade de vaso para a a produção de mudas de faveleria, por proporcionar maior crescimento.
\end{abstract}

Palavras-chave: Cnidoscolus quercifolius, produção de mudas, estresse hídrico, rejeito de mineração.

\section{Growth and seedlings quality of Cnidoscolus quercifolius Pohl in relation to substrate and water levels}

\begin{abstract}
In view of the Brazilian ecosystems degradation, it is necessary to produce quality seedlings in order to recover them. However, to meet this purpose it is necessary to cause the minimum possible environmental impact. In this sense, the use of alternative materials in the composition of the substrate, as well as reduction in the amount of water in the nursery phase are aspects that must be considered. The present work was conducted with the objective of evaluating substrates and water levels in the growth and quality of faveleira seedlings, four mixtures were evaluated, two being between soil and manure (2: 1 and 3:1) and soil and vermiculite tailings (2: 1 and 3: 1) combined with four water levels $(100,75,50$ and $25 \%$ of pot capacity) in the $4 \times 4$ factorial scheme. The treatments were distributed in a completely randomized design with four replicates per treatment. The parameters analyzed were plant height, stem diameter; dry masses of shoots, roots, leaves, and the total; absolute growth rate; the height/diameter ratio and the Dickson's Index Quality. The substrate composed of soil + bovine manure provides the production of better quality seedlings. The water level between $75 \%$ and $100 \%$ of the pot capacity is recommended for the production of faveleria seedlings, as it provides greater growth. Water levels below $75 \%$ of pot capacityCP reduce plant growth and dry matter. The substrates soil+bovine manure and soil+vermiculite tailings, in the 2:1 proportion provide a better quality of seedlings.
\end{abstract}

Keywords: seedlings production, water stress, mining co-product.

\footnotetext{
${ }^{1}$ Submetido em 19/10/2018 e aprovado em 23/04/2019

${ }^{2}$ Engenheiro Florestal; Estudante de Mestrado, Universidade Federal de Campina Grande (UFCG), Unidade Acadêmica de Engenharia Florestal, Programa de Pós-Graduação em Ciências Florestais, Patos-Paraíba, CEP: 58.708-110; E-mail: rosilvam17@ gmail.com

${ }^{3}$ Doutor em Agronomia; Professor Titular, Universidade Federal de Campina Grande (UFCG), Unidade Acadêmica de Engenharia Florestal (UAEF), Patos-Paraíba, CEP: 58.708-110; E-mail: lucineudofreire@gmail.com
} 


\section{Introdução}

A vegetação do tipo Caatinga é constituída de arbustos e árvores, reflexo das condições edaficlimáticas predominantes, com chuvas irregulares, espacial e temporalmente, concentradas em quatro meses do ano, com baixa umidade e alta temperatura atmosférica (Lepsch, 2002). Em virtude da distribuição irregular da precipitação e da presença de solos rasos e/ou salinos, em algumas regiões, a capacidade de armazenamento é limitante.

No Nordeste brasileiro, os recursos madeireiros têm sido utilizados principalmente para produção de lenha e carvão, abastecendo olarias, padarias, cerâmicas e para o consumo do homem do campo (Rodrigues et al., 2014). Em virtude dessa exploração, é urgente a necessidade de repor a vegetação retirada e recompor as áreas degradadas, visando minimizar os impactos ambientais e a manutenção da biodiversidade. Esse fato tem proporcionado aumento na procura por mudas de espécies nativas. É imperativo então que se produza mudas de qualidade para atender a essas demandas, garantindo assim a adaptação e o crescimento das mesmas após o plantio no campo (Kratka e Correia, 2015).

A qualidade de uma muda é resultante da interação de vários fatores bióticos e abióticos, sendo que o substrato assume grande importância no sistema de produção de mudas (Martins et al., 2011). O substrato ideal deve proporcionar condições adequadas à germinação e ao desenvolvimento inicial das mudas, apresentando boas características químicas e físicas como densidade, capacidade de retenção de água, aeração e drenagem adequadas, de modo a evitar acúmulo de umidade (Pio et al., 2005; Cunha et al., 2006), além de estar isento de patógenos, plantas daninhas (Dantas et al., 2009) e substâncias tóxicas (Pio et al., 2005).

A crescente necessidade de se produzir mudas de qualidade, em curto período de tempo, e ocupando pouco espaço, tem exigido a busca por alternativas para que essa produção seja rápida e sustentável, sem agredir o meio ambiente. Vários são os produtos utilizados na composição de substratos para a produção de mudas de espécies agrícolas e arbóreas, a exemplo de vermicomposto (Steffen et al., 2011), casca de côco (Rosa et al., 2002; Lacerda et al., 2006), casca de arroz carbonizada (Vidal et al., 2006;
Caldeira et al., 2015), acícula de pinus, lodo de esgoto (Kratka e Correia, 2015; Caldeira et al., 2015), bagana de carnaúba (Araújo et al., 2017). Os materiais a serem empregados devem ser de fácil acessibilidade na região do estudo, de forma a reduzir os custo de produção, além de diminuir o impacto ambiental que determinados materiais ocasionam, quando depositados no meio ambiente (Toledo et al., 2015; Araújo et al., 2017), a exemplo do rejeito de vermiculita, um co-produto da extração desse minério, na região semiárida da Paraíba (Trajano et al., 2010; Rodrigues et al., 2014).

Além do substrato, outro fator relevante na produção de mudas é a qualidade e a quantidade de água disponibilizada. A irrigação em viveiros florestais, principalmente durante a fase de rustificação, é feita de uma forma bastante empírica, onde apenas o exame visual determina o momento e a conduta de irrigação a ser tomada (tempo e frequência) (Lopes et al., 2005; Simões et al., 2012).

Pertencente à família Euphorbiaceae, a faveleira (Cnidoscolus quercifolius Pohl.), espécie nativa das caatingas do Nordeste, xerófila, decídua, de uso múltiplo, podendo ser empregada para recuperação de áreas degradadas, alimentação animal e humana, medicina, serraria e energia, biodiesel, dentre outros (Lorenzi, 2000; Arriel et al., 2004; Maia, 2004).

$\mathrm{O}$ conhecimento a respeito de aspectos relacionados à produção de mudas de espécies nativas da Caatinga é escasso e, apesar da sua importância econômica, pouco se sabe a respeito dos aspectos relacionados à produção de mudas da faveleira. Tais informações são necessárias para se obter sucesso em programas de revegetação e/ou recuperação de áreas degradadas.

Dessa forma, o presente estudo foi realizado com o objetivo avaliar o crescimento e a qualidade das mudas de faveleira produzidas em substratos e volumes de irrigação.

\section{Material e Métodos}

$\mathrm{O}$ experimento foi conduzido em ambiente telado (7'03'34.83's e $37^{\circ} 16^{\prime} 28.40^{\prime}$ 'O, altitude $253 \mathrm{~m})$, localizado no Viveiro Florestal da Unidade Acadêmica de Engenharia Florestal, pertencente ao Centro de Saúde e Tecnologia 
Rural da Universidade Federal de Campina Grande (CSTR/UFCG), Patos - PB.

Antes da semeadura, as sementes de faveleira foram submetidas à quebra da dormência mecânica do tegumento com auxílio de lixa. Em seguida, foram colocadas para germinar em bandejas plásticas contendo areia e aos 10 dias após a emergência, transferidas para sacos plásticos pretos $(30 \mathrm{~cm} \times 12 \mathrm{~cm})$ contendo $5 \mathrm{~kg}$ do substrato, de acordo com o tratamento.
Na preparação dos substratos utilizou-se terra de subsolo, coletada na Fazenda Nupeárido (Núcleo de Pesquisa do Semiárido) (CSTR/UFCG). De acordo com a análise física, o solo componente dos substratos possuía 780, 100 e $120 \mathrm{~g} \mathrm{~kg}^{-1}$ de areia, silte e argila, respectivamente, com classificação textural areia franca. O rejeito de vermiculita foi adquirido na empresa UBM, em Santa Luzia-PB. Ambos os materiais foram submetidos à análise de fertilidade (Tabela 1), empregando-se a metodologia proposta pela Embrapa (1997).

Tabela 1 Análises químicas do solo e do rejeito de vermiculita (RV) utilizados no experimento

\begin{tabular}{|c|c|c|c|c|c|c|c|c|c|}
\hline \multirow{2}{*}{ Material } & \multirow{2}{*}{$\begin{array}{c}\mathrm{pH} 1: 2,5 \\
\left(\mathrm{CaCl}_{2} 0,01 \mathrm{M}\right)\end{array}$} & \multirow{2}{*}{$\begin{array}{c}\mathrm{P} \\
\mathrm{g} \mathrm{dm}^{-3} \\
\end{array}$} & $\mathrm{~K}$ & $\mathrm{Na}$ & $\mathrm{Ca}$ & $\mathrm{Mg}$ & $\mathrm{H}+\mathrm{Al}$ & $\mathrm{T}$ & \multirow{2}{*}{$\begin{array}{l}\mathrm{V} \\
\% \\
\end{array}$} \\
\hline & & & \multicolumn{6}{|c|}{ 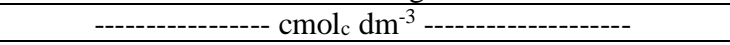 } & \\
\hline Solo & 5,0 & 0,045 & 0,17 & 0,57 & 5,10 & 1,90 & 3,10 & 10,84 & 71,4 \\
\hline RV & 6,8 & 0,040 & 0,20 & 2,00 & 3,80 & 2,40 & 1,00 & 9,60 & 89,0 \\
\hline
\end{tabular}

Após o enchimento dos sacos, foi determinada a capacidade de vaso, calculada segundo metodologia descrita por Souza et al. (2000), a partir do qual foram determinados os níveis de água a serem avaliados.

Os tratamentos foram distribuídos em delineamento inteiramente casualizado (DIC), em esquema fatorial $4 \times 4$, sendo quatro substratos [solo + esterco bovino (2:1), solo + esterco bovino (3:1), solo + rejeito de vermiculita (2:1) e solo + rejeito de vermiculita (3:1)] e quatro níveis de água $[100,75,50$ e $25 \%$ da capacidade de vaso (cv)] e quatro repetições, sendo que cada parcela era composta por cinco plantas, totalizando 320 plantas.

Durante os 15 primeiros dias após o transplantio das plântulas para os sacos plásticos, foi feita a aclimatação das mudas, mantendo-se o substrato com nível de umidade de $100 \%$ cv. Decorrido esse período, foi feita a diferenciação dos tratamentos hídricos.

A superfície superior dos recipientes foi coberta com um plástico opaco, no intuito de minimizar a perda de água do substrato por evaporação. Para manter os níveis de água estabelecidos, a água perdida por transpiração foi reposta diariamente através da pesagem dos vasos, com auxílio de balança, de acordo com cada tratamento de irrigação.

No início da diferenciação dos tratamentos hídricos, foi feita a medição da altura (altura inicial Ai). Aos 150 dias após início dos tratamentos hídricos (DAIT), o experimento foi encerrado e foram avaliadas a altura final das plantas (A), diâmetro do caule (D), tomado ao nível do substrato, massa seca das folhas (MSF), do caule (MSC), da parte aérea (MSPA), das raízes (MSR) e massa seca total (MSTt).

De posse dos dados, foram calculadas a taxa de crescimento absoluto (TCA) (BENINCASA, 2003) e a razão altura/diâmetro, respectivamente através das equações $\mathrm{TCA}=(\mathrm{Af}-\mathrm{Ai}) / \Delta \mathrm{t}$ e RAD $=\mathrm{A} / \mathrm{D}$, em que Af e Ai correspondem às alturas aos 150 e 0 DAIT, $\Delta \mathrm{t}$ o intervalo de tempo entre as medições e $\mathrm{D}$, ao diâmetro do caule.

A qualidade das mudas foi avaliada pelo Índice de Qualidade de Dickson (IQD) (DICKSON et al., 1960), através da equação IQD $=$ MSTt $/ \mathrm{RAD}+\mathrm{RPAR}$, sendo que MSTt e RPAR correspondem, respectivamente, à massa seca total e razão massa seca da parte aérea/massa seca das raízes, respectivamente.

Os dados foram submetidos à análise de variância, sendo as médias dos substratos comparadas pelo teste de Tukey a $5 \%$ de probabilidade de erro, e aplicada a análise de regressão nos tratamentos de nível de água, quando necessário, utilizando-se o software ASSISTAT versão 7.7 (SILVA e AZEVEDO, 2002).

\section{Resultados e Discussão}

De acordo com a análise de variância (Tabela 2), os níveis de água afetaram significativamente 
todos os parâmetros analisados, exceto o diâmetro do caule e a massa seca das folhas, enquanto que os substratos exerceram efeito significativo nos parâmetros avaliados, exceto altura de plantas,
Taxa de crescimento absoluto (TCA) e razão altura/diâmetro (RAD). Não foi verificada interação significativa entre os fatores sobre os parâmetros analisados.

Tabela 2 Resumo das análises de variância de altura de plantas (A), diâmetro do coleto (D), massa seca do caule (MSC), massa seca de folhas (MSF), massa seca da parte aérea (MSPA), massa seca das raízes (MSR), massa seca total (MSTt), razão altura/diâmetro (RAD), taxa de crescimento absoluto (TCA) e Índice de Qualidade de Dickson (IQD)

\begin{tabular}{|c|c|c|c|c|c|c|}
\hline \multirow[t]{2}{*}{ Fonte de variação } & \multirow[t]{2}{*}{ GL } & \multicolumn{5}{|c|}{ Quadrados médios } \\
\hline & & $\mathbf{A}$ & D & MSC & MSF & MSPA \\
\hline Níveis de água (A) & 3 & $7359,2350^{* *}$ & $5,2606^{\mathrm{ns}}$ & $25,8702^{*}$ & $5,7449^{\text {ns }}$ & $651,6474^{*}$ \\
\hline Substrato $(\mathrm{S})$ & 3 & $238,9829^{\text {ns }}$ & $60,0418^{* *}$ & $251,6629^{* *}$ & $143,6601^{* *}$ & $769,5226^{* *}$ \\
\hline $\mathrm{N} \times \mathrm{S}$ & 9 & $117,8143^{\text {ns }}$ & $2,8908^{\mathrm{ns}}$ & $9,1651^{\mathrm{ns}}$ & $4,2879^{\mathrm{ns}}$ & $24,3981^{\mathrm{ns}}$ \\
\hline Resíduo & & 135,0174 & 1,9048 & 8,6367 & 3,5509 & 21,0583 \\
\hline $\mathrm{CV}(\%)$ & & 37,98 & 16,08 & 62,32 & 34,29 & 44,94 \\
\hline \multirow{2}{*}{ Fonte de variação } & GL & \multicolumn{5}{|c|}{ Quadrados médios } \\
\hline & & MSR & MSTt & RAD & TCA & IQD \\
\hline Níveis de água (A) & 3 & $2232,2828^{* *}$ & $2932,2321^{* * *}$ & $51,0828^{* * *}$ & $0,3270^{* *}$ & $226,3233^{*}$ \\
\hline Substrato $(S)$ & 3 & $1136,8443^{*}$ & $2508,8915^{* *}$ & $0,6621^{\mathrm{ns}}$ & $0,0106^{\mathrm{ns}}$ & $219,6005^{*}$ \\
\hline $\mathrm{N} \times \mathrm{S}$ & 9 & $421,9068^{\mathrm{ns}}$ & $576,3681^{\mathrm{ns}}$ & $0,9314^{\mathrm{ns}}$ & $0,0052^{\mathrm{ns}}$ & $68,0461^{\mathrm{ns}}$ \\
\hline Resíduo & & 332,047 & 375,9413 & 0,9569 & 0,0061 & 78,2559 \\
\hline $\mathrm{CV}(\%)$ & & 53,87 & 43,98 & 30,36 & 41,22 & 61,83 \\
\hline
\end{tabular}

\subsection{Substrato}

Os substratos que apresentaram esterco bovino (S:E 2:1 e S:E 3:1) na sua composição proporcionaram os maiores valores de diâmetro do caule e acúmulo de biomassa do caule, das folhas e da parte aérea (Tabela 3). Quando se compara os substratos solo:esterco bovino $2: 1 \mathrm{e}$ solo:rejeito de vermiculita $2: 1$, nota-se redução de $78 \%, 50 \%$ e $65 \%$ nos valores de MSC, MSF e MSPA. Além disso, constata-se que a diminuição no acúmulo de biomassa nesses componentes foi mais acentuada quando a comparação é feita com o substrato solo:rejeito de vermiculita 3:1.

Tabela 3 Diâmetro do caule (D), massa seca do caule (MSC), das folhas (MSF), da parte aérea (MSPA), das raízes (MSR) e total (MSTt), razão massa seca das raízes/massa seca da parte aérea (R/PA) e Índice de Qualidade de Dickson (IQD) das plantas de faveleira em função dos substratos

\begin{tabular}{|c|c|c|c|c|c|c|c|c|}
\hline \multirow[t]{2}{*}{ Substratos* } & $\mathrm{D}(\mathrm{mm})$ & MSC & MSF & MSPA & MSR & MSTt & \multirow[t]{2}{*}{$\mathrm{R} / \mathrm{PA}$} & \multirow[t]{2}{*}{ IQD } \\
\hline & \multicolumn{6}{|c|}{ (g) } & & \\
\hline $\mathrm{S}: \mathrm{E}(2: 1)$ & $9,8 \mathrm{a}$ & $8,8 \mathrm{a}$ & $8,3 \mathrm{a}$ & $17,1 \mathrm{a}$ & $41,8 \mathrm{a}$ & $58,9 \mathrm{a}$ & $2,44 \mathrm{~b}$ & $17,5 \mathrm{a}$ \\
\hline $\mathrm{S}: \mathrm{E}(3: 1)$ & $10,5 \mathrm{a}$ & $7,4 \mathrm{a}$ & $7,6 \mathrm{a}$ & $15,0 \mathrm{a}$ & $27,5 \mathrm{~b}$ & $42,5 \mathrm{ab}$ & $1,83 \mathrm{~b}$ & $10,7 \mathrm{~b}$ \\
\hline $\mathrm{S}: \mathrm{RV}(2: 1)$ & $7,8 \mathrm{~b}$ & $1,9 \mathrm{~b}$ & $4,1 \mathrm{~b}$ & $5,9 \mathrm{~b}$ & $40,5 \mathrm{a}$ & $46,4 \mathrm{ab}$ & $6,86 \mathrm{a}$ & $17,6 \mathrm{a}$ \\
\hline S:RV $(3: 1)$ & $6,2 \mathrm{c}$ & $0,8 \mathrm{~b}$ & $2,0 \mathrm{c}$ & $2,8 \mathrm{~b}$ & $25,7 \mathrm{~b}$ & $28,5 \mathrm{~b}$ & $9,18 \mathrm{a}$ & $11,5 \mathrm{~b}$ \\
\hline
\end{tabular}

Médias seguidas de letras iguais não diferem estatisticamente entre si pelo teste de Tukey $(\mathrm{p} \leq 0,05)$. *S:E - solo: esterco bovino; S:RV - solo: rejeito de vermiculita.

Em relação à biomassa das raízes (MSR), os maiores valores foram obtidos nos tratamentos S:E 2:1 e S:RV 2:1, ou seja, naqueles em que o solo foi empregado em menor proporção em relação ao outro componente do substrato (esterco bovino ou rejeito de vermiculita) (Tabela $3)$.

Analisando-se a razão R/PA, constata-se que os substratos que receberam rejeito de vermiculita apresentaram os maiores valores (Tabela 3). Quanto à massa seca total, o substrato $\mathrm{S}: \mathrm{E}$ 2:1 possibilitou maior produção que os demais, apesar de ter sido verificada igualdade estatística com os substratos S:E 3:1 e S:RV 2:1 (Tabela 3). Em decorrência dos valores de MSR e MSTt obtidos, os substratos S:E 2:1 e S:RV 2:1 possibilitaram maior IQD (Tabela 3).

Uma vez que o solo utilizado nesse estudo era de classificação textural areia franca, possivelmente a adição de esterco bovino e do rejeito de vermiculita em maior proporção $(2: 1)$ tenha proporcionado maior retenção de umidade, 
favorecendo maior acúmulo de massa seca na parte aérea, massa seca total e qualidade das mudas.

O uso do rejeito de vermiculita na composição do substrato para a faveleira (Cnidoscolus quercifolius Pohl.) foi recomendado por Trajano et al. (2010) como forma de reduzir o impacto do seu acúmulo no meio ambiente. Rodrigues et al. (2014) e Silva et al. (2014) indicam esse produto em adição à matéria orgânica na produção de mudas de Tabebuia caraiba e Mimosa caesalpiniifolia. Igualmente, Leite et al. (2015) recomendam o uso de rejeito de vermiculita em áreas salinizadas do Cariri Paraibano, visando reduzir os efeitos da salinidade no cultivo de maracujazeiro (Passiflora edulis).

$\mathrm{Na}$ composição do substrato para enraizamento de estacas de faveleira, Farias Júnior et al. (2015) verificaram que o substrato rejeito de vermiculita teve um desempenho superior em todas as variáveis estudadas, influenciando significativamente a produção de massa seca radicular das estacas de faveleira, evidenciando sua grande importância como substrato alternativo para a produção de mudas, sobretudo pela fácil aquisição e baixo custo.

\subsection{Níveis de água}

Diminuição nos níveis de água promoveu redução linear na altura das mudas (Figura 1). Comparando-se as mudas do tratamento controle $(100 \% \mathrm{cv}) \mathrm{com}$ as do tratamento $50 \% \mathrm{cv}$, verificase redução de $70 \%$ na altura das plantas. Essa diminuição chega a $82 \%$ quando a comparação é feita com o tratamento $25 \% \mathrm{cv}$.

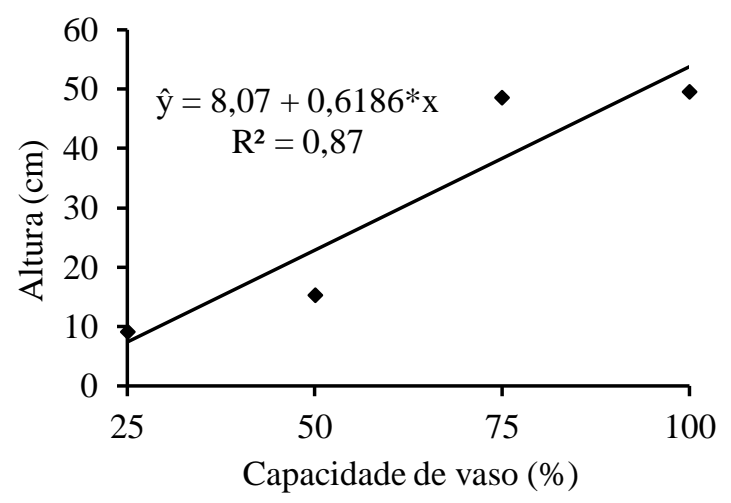

Figura 1 Altura de mudas de faveleira em função dos níveis de água

A redução no crescimento em altura pode ser decorrente da menor taxa de alongamento, diferenciação e divisão celular, causadas pela diminuição da turgescência sob condições de baixa disponibilidade hídrica (Hsiao e Xu, 2000; Nogueira et al., 2005). Além disso, a diminuição no teor de água do solo promove redução no potencial hídrico foliar, causando fechamento total ou parcial dos estômatos, comprometendo o influxo de $\mathrm{CO}_{2}$ e consequentemente a fotossíntese e a produção de assimilados (Chaves et al., 2002). Outro fato que deve ser considerado é que sob condições de déficit hídrico ocorre diminuição na absorção de nutrientes (Taiz e Zeiger, 2013). Tais aspectos podem causar redução na área foliar (Bergamaschi et al., 2009), afetando, dessa forma, a produção e a translocação de fotoassimilados para as regiões de crescimento (Larcher, 2004; Taiz e Zeiger, 2013). Redução na altura das plantas em decorrência da diminuição na quantidade de água fornecida também foi verificada em Myracrodruon urundeuva (Figueirôa et al., 2004), Guazuma ulmiflora (Scalon et al., 2011) e Jatropha curcas (Padilha et al., 2016).

Quanto à produção de massa seca do caule (Figura 2A), verificou-se decréscimo nos valores à medida que a quantidade de água fornecida ao substrato diminuía, ocorrendo redução de 0,0392 g para cada redução de $1 \%$ da umidade do solo. Nos outros componentes das plantas, percebe-se que os níveis de redução para cada $1 \%$ de umidade do solo foram mais altos, chegando a $0,0544 \mathrm{~g}, 0,3632 \mathrm{~g}$ e $0,4156 \mathrm{~g}$, respectivamente para MSPA (Figura 2B), MSR (Figura 2C) e MSTt (Figura 2D).

Comparando-se as plantas do tratamento que recebeu maior quantidade de água $(100 \% \mathrm{cv}) \mathrm{com}$ aquelas do tratamento $25 \% \mathrm{cv}$, observaram-se reduções, respectivamente, de $48 \%, 35 \%, 58 \%$ e $54 \%$, em MSC, MSPA, MSR e MSTt. Percebese, então, que a maior redução ocorreu na matéria seca das raízes, mostrando que este compartimento das plantas de faveleira foi mais sensível, contrariamente ao que acontece com muitas espécies sob condições de baixa disponibilidade de água (Taiz e Zeiger, 2013). Barbosa et al. (2000), avaliando os efeitos da suspensão da irrigação em plantas jovens de Senna martiana, espécie lenhosa da Caatinga, verificaram que as mesmas alocaram mais assimilados nas folhas. Comportamento semelhante foi verificado por Cabral et al. (2004), em Tabebuia aurea. 

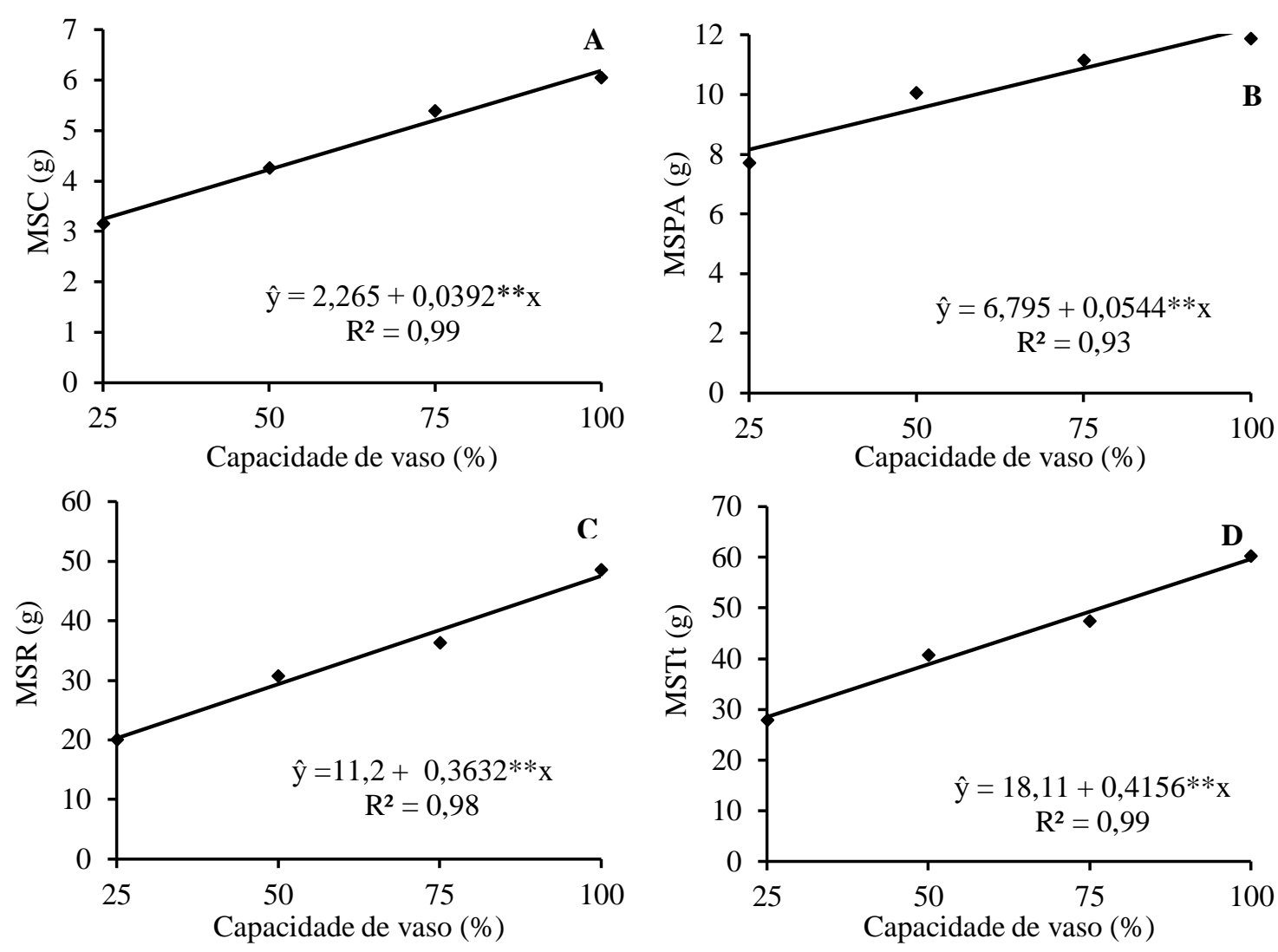

Figura 2. Massa seca do caule (A), parte aérea (B), raizes (C) e total (D) de mudas de faveleira em função da umidade do substrato.

Esse comportamento diferiu dos resultados obtidos por Figueirôa et al. (2004), os quais verificaram que o estresse hídrico proporcionou aumento na alocação de biomassa das raízes de Myracrodruon urundeuva, 30 dias após o início dos regimes hídricos. Esses autores relataram ainda que não houve diferença estatística na biomassa das raízes, quando a avaliação foi realizada aos 60 dias.

Ao final do experimento, independente da nível irrigação, as plantas apresentaram maior massa seca nas raízes do que na parte aérea. Esse comportamento deve-se ao fato da espécie possuir raízes tuberosas, na fase de muda, possibilitando o armazenamento de reservas, garantindo a manutenção das atividades vitais da planta durante a seca (Maia, 2004) e permitindo o surgimento de novas folhas após a retormada das chuvas aparecimento de que, sob condições de baixa disponibilidade de água, as plantas. O maior direcionamento de assimilados para as raízes, de acordo com Sanford e Cuevas (1996), constituise em uma estratégia de adaptação à condição de menor disponibilidade de água.
Em relação à TCA e RAD (Tabela 4), percebese que os tratamentos 100 e $75 \%$ cv proporcionaram os maiores valores, sendo superiores aos demais tratamentos. Nos tratamentos 50 e $25 \% \mathrm{cv}$ a TCA foi 4 e 6 vezes, respectivamente, menor que os tratamentos $100 \mathrm{e}$ $75 \%$ cv. Quanto à RAD, os valores obtidos nas plantas submetidas à maior disponibilidade de água $(100 \% \mathrm{cv})$ corresponderam a 2,5 e 4,5 vezes maiores que os obtidos nas plantas dos tratamentos 50 e $25 \% \mathrm{cv}$, respectivamente.

Em plantas de Azadirachta indica, Martins et al. (2010) verificaram que sob baixa disponibilidade hídrica ocorreu redução na matéria seca das plantas. Comportamento semelhante foi verificado em plantas de Prosopis argentina e Prosopis alpataco, por Villagra e Cavagnaro (2006), os quais constataram reduções na matéria seca em todas as partes das plantas, assim como na matéria seca total. Cabral et al. (2004) observaram que até os 120 dias de idade, as plantas de Tabebuia aurea mantidas a $25 \%$ da capacidade de campo (cc) apresentaram redução no desenvolvimento da parte aérea. 
Tabela 4 Taxa de crescimento absoluto (TCA), razão entre altura e diâmetro (RAD) e índice de qualidade de Dickson (IQD) de mudas de faveleira em função da umidade do substrato

\begin{tabular}{lccc}
\hline $\begin{array}{c}\text { Níveis umidade } \\
(\% \mathbf{~ c v})\end{array}$ & $\begin{array}{c}\text { TCA } \\
\left(\mathbf{c m ~ d i a}^{-1}\right)\end{array}$ & RAD & IQD \\
\hline 25 & $0,05 \mathrm{~b}$ & $1,45 \mathrm{~b}$ & $15,3 \mathrm{~b}$ \\
50 & $0,08 \mathrm{~b}$ & $1,94 \mathrm{~b}$ & $19,1 \mathrm{a}$ \\
75 & $0,31 \mathrm{a}$ & $4,91 \mathrm{a}$ & $11,1 \mathrm{c}$ \\
100 & $0,32 \mathrm{a}$ & $4,99 \mathrm{a}$ & $11,5 \mathrm{c}$ \\
\hline \multicolumn{4}{l}{ Médias seguidas de letras iguais não diferem estatisticamente entre } \\
si pelo teste de Tukey $(\mathrm{p} \leq 0,05)$.
\end{tabular}

Segundo Hsiao e Xu (2000) e Chaves et al. (2002), o primeiro processo a ser afetado, e o mais sensível, é a expansão e o crescimento celular, em decorrência da diminuição do volume e da turgescência das células. Esses fatores levam à redução no crescimento e consequente acúmulo de biomassa nas plantas. Dentre as alterações que ocorrem sob condições de déficit hídrico está o fechamento estomático, reduzindo a transpiração (Chaves et al., 2002; Gomes et al., 2004; Silva et al., 2004) e a condutância estomática (Nogueira e Silva, 2002; Queiroz et al., 2002; Gomes et al., 2004) que limita a difusão do $\mathrm{CO}_{2}$ para os cloroplastos, interferindo diretamente na capacidade fotossintética da plantas e, consequentemente, no acúmulo de biomassa e crescimento das plantas (Chaves et al., 2002). Outro fator que deve ser considerado é a redução na atividade e ativação da enzima Rubisco causado pelo déficit hídrico (Medrano et al., 2002), concorrendo para a prejuízos na fotossíntese.

\section{Conclusão}

Substrato composto solo + esterco bovino proporciona maior crescimento e qualidade de mudas de faveleira;

Recomenda-se o nível de água entre 75 e $100 \%$ da capacidade de vaso, para a produção de mudas de faveleira por proporcionar bom crescimento.

\section{Referências}

Arriel, E. F.; Paula, R. C.; Bakke, O. A.; Arriel, N. H. C. Divergência genética em Cnidoscolus phyllacanthus (Mart.) Pax. Et K. Hoffm. Revista Brasileira de Oleaginosas Fibrosas, v.8, n.2/3, p.813-822, 2004.

Barbosa, D. C. A.; Nogueira, R. J. M. C.; Melo Filho, P. A. Comparative studies of growth in three species of caatinga submitted to water stress. Phyton, v.69, n.1, p.45-50, 2000.

Benincasa, M. M. P. Análise de crescimento de plantas. 2 ed. Jaboticabal: FUNEP, 2003. 41 p.

Bergamaschi, H.; Dalmago, G. A.; Bergonci, J. I.; Bianchi, C. A. M.; Müller, A. G.; Comiran, F.; Heckler, B. M. M. Distribuição hídrica no período crítico do milho e produção de grãos. Pesquisa Agropecuária Brasileira, v.39, n.9, p.831-839, 2004. http://www.scielo.br/pdf/pab/v39n9/22025.pdf

Cabral, E. L.; Barbosa, D. C. A.; Simabukuro, E. A. Crescimento de plantas jovens de Tabebuia aurea (Manso) Benth. \& Hook. F. ex S. Moore submetidas a estresse hídrico. Acta Botanica Brasilica, v.18, n.2, p.241-251, 2004. http://dx.doi.org/10.1590/S0102-33062004000200004

Caldeira, M. V. W.; Delarmelina, W. M.; Faria, J. C. T.; Juvanhol, R. S. Substratos alternativos na produção de mudas de Chamaecrista desvauxii L. Revista Árvore, v.37, n.1, p.31-39, 2013. http://www.scielo.br/pdf/rarv/v37n1/v37n1a04.pdf

Chaves, M. M.; Pereira, J. S.; Maroco, J.; Rodrigues, M. L.; Ricardo, C. P. P.; Osório, M. L.; Carvalho, I.; Faria, T.; Pinheiro, C. How plants cope with water stress in the field? Photosynthesis and growth. Annals of Botany, v.89, n.1, p.907-916, 2002. https://doi.org/10.1093/aob/mcf105

Cunha, A. M.; Cunha, G. M.; Sarmento, R. A.; Cunha, G. M.; Amaral, J. F. T. Efeito de diferentes substratos sobre o desenvolvimento de mudas de Acacia sp. Revista Árvore, v.30, n.2, p.207-214, 2006. http://www.scielo.br/pdf/\%0D/rarv/v30n2/a07v30n2.pdf

Dantas, B. F.; Lopes, A. P.; Silva, F. F. S.; Lúcio, A. A.; Batista, P. F.; Pires, M. M. M. L.; Aragão, C. A. Taxas de crescimento de mudas de catingueira submetidas a diferentes substratos e sombreamentos. Revista Árvore, v.33, n.3, p.413-423, 2009. https://www.alice.cnptia.embrapa.br/handle/doc/577693

Dickson, A.; Leaf, A. L.; Hosner, J. F. Quality appraisal of white spruce and white pine seedling stock in nurseries. Forestry Chronicle, v.36, p.10-13, 1960. https://doi.org/10.5558/tfc36010-1

Embrapa. Empresa Brasileira de Pesquisa Agropecuária. Manual de métodos de análise 
de solo. Rio de Janeiro: Ministério da Agricultura, 1997.

Farias Júnior, J. A.; Arriel, E. F.; Lúcio, A. M. F. N.; Freire, A. L. O.; Santos, R. V.; Lucena, R. J. Clonagem de Cnidoscolus quercifolius por alporquia, utilizando rejeito de vermiculita e diferentes concentrações de AIA. Pesquisa Florestal Brasileira, v.35, n.81, p.35-40, 2015. https://doi.org/10.4336/2015.pfb.35.81.499

Figueirôa, J. M.; Barbosa, D. C. A.; Simabukuro, E. A. Crescimento de plantas jovens de Myracrodruon urundeuva Allemão (Anacardiaceae) sob diferentes regimes hídricos. Acta Botanica Brasilica, v.18, n.3, p.573-580, 2004. http://agris.fao.org/agrissearch/search.do?recordID=XS2010100433

Gomes, M. M. A.; Lagôa, A. M. M. A.; Medina, C. L.; Machado, E. C.; Machado, M. A. Interactions between leaf water potential, stomatal conductance and abscisic acid content of orange trees submitted to drought stress. Brazilian Journal of Plant Physiology, v.16, n.3, p.155-161, 2004. http://dx.doi.org/10.1590/S1677-04202004000300005

Gonçalves, J. L. M.; Santerelli, E. G.; Moraes Neto, S. P.; Manara, M. P. Produção de mudas de espécies nativas: substrato, nutrição, sombreamento e fertilização. IN: Gonçalves, J. L. M.; Benedetti, V. (Eds.). Nutrição e fertilização florestal. Piracicaba: ESALQ/USP, 2000. p. 309-350.

Hsiao, T. C.; Xu, L. K. Sensitivity of growth of roots versus leaves to water stress: biophysical analysis and relation to water transport. Journal of Experimental Botany, v.51, n.350, p.15951616, 2000. https://doi.org/10.1093/jexbot/51.350.1595

Larcher, W. Ecofisiologia Vegetal. São Carlos: Editora RIMA, 2004.

Leite, M. J. H.; Gomes, A. D. V.; Santos, R. V. Comportamento inicial do maracujazeiro em solos afetados por sais submetidos a tratamentos alternativos com o uso de coprodutos de mineradoras. Irriga, v.20, n.3, p.401-413, 2015. https://doi.org/10.15809/irriga.2015v20n3p401

Lepsch, I. F. Formação e Conservação dos Solos. São Paulo: Oficina de textos, 2002. 178p.

Lorenzi, H. Árvores brasileiras: manual de identificação e cultivo de plantas arbóreas do Brasil. 3. ed. Nova Odessa: Instituto Plantarum, 2000. 373p.
Maia, G. N. Caatinga: árvores e arbustos e suas utilidades. 1 ed. São Paulo: D\&Z Computação Gráfica e Editora, 2004. 413 p.

Martins, C. C.; Machado, C. G.; Caldas, I. G. R.; Vieira, I. G. Vermiculita como substrato para o teste de germinação de sementes de barbatimão. Ciência Florestal, v.21, n.3, p.421-427, 2011. http://dx.doi.org/10.5902/198050983800

Martins, M. O.; Nogueira, R. J. M. C.; Azevedo Neto, A. D.; Santos, M. G. Crescimento de plantas jovens de nim-indiano (Azadirachta indica A. Juss - Meliaceae) sob diferentes regimes hídricos. Revista Árvore, v.34, n.5, p.771-779, 2010 . https://www.redalyc.org/html/488/48815860002/

Medrano, H.; Escalona, J. M.; Bota, J.; Gulías, J.; Flexas, J. Regulation of photosynthesis of C3 plants in response to progressive drought: stomatal conductance as a reference parameter. Annals of Botany, v.89, n.4, p.895-905, 2002. https://www.redalyc.org/html/488/48815860002/

Nogueira, R. J. M. C.; Albuquerque, M. B.; Silva, E. C. Aspectos ecofisiológicos da tolerância à seca em plantas da Caatinga. In: Nogueira, R. J. M. C.; Araújo, E. L.; Willadino, L. G.; Cavalcante, U. M. T. Estresses ambientais: danos e benefícios em plantas. Recife: Universidade Federal de Pernambuco, 2005. p. 22-31.

Nogueira, R. J. M. C.; Silva, E. C. Comportamento estomático em plantas jovens de Schinopsis brasiliensis Engl. cultivadas sob estresse hídrico. Iheringia: Série Botânica, v.57, n.1, p.31- 38, 2002.

Padilha, N. S.; Silva, C. J.; Pereira, S. B.; Silva, J. A. N.; Heid, D. M.; Bottega, S. P.; Scalon, S. P. Q. Crescimento inicial do pinhã-manso submetido a diferentes regimes hídricos em latossolo vermelho distrófico. Ciência Florestal, v.26, n.2, p.513-521, 2016. http://dx.doi.org/10.5902/1980509822752

Pio, R.; Ramos, J. D.; Gontijo, T. C. A.; Carrijo, E. P.; mendonça, V. ; Fabri, E. G.; Chagas, E. A. Substratos na produção de mudas de jabuticaba. Revista Brasileira de Agrociência, v.11, n.4, p.425-427, 2005. http://dx.doi.org/10.18539/cast.v11i4.1280

Queiroz, C. G. S.; Garcia, Q. S.; Lemos Filho, J. P. Atividade fotossintética e peroxidação de lipídios de membrana em plantas de aroeira-do- 
sertão sob estresse hídrico e após reidratação. Brazilian Journal of Plant Physiology, v.14, n.1, p.59-63, 2002. http://www.scielo.br/pdf/\%0D/rbfv/v14n1/11784.pdf

Rodrigues, R. D.; Freire, A. L. O.; Nascimento Neto, J. H. Uso de rejeitos de mineração e materiais orgânicos na composição de substrato para a produção de mudas de sabiá. Engenharia Ambiental, v.11, n.1, p.16-27, 2014. http://ferramentas.unipinhal.edu.br/engenhariaambiental/v iewarticle.php?id=914

Rosa, M. F.; Bezerra, F. C.; Correia, D.; Santos, F. J. S.; Abreu, F. A. P.; Furtado, A. A. L.; Brígido, A. K. L.; Norões, E. R. V. Utilização da casca de coco como substrato agrícola. Fortaleza: Embrapa Agroindústria Tropical, 2002. 24p. (Embrapa Agroindústria Tropical. Documentos, 52). https://www.infoteca.cnptia.embrapa.br/bitstream/doc/42 5175/1/doc52.pdf

Rubira, J. L. P., Bueno, L. O. Cultivo de plantas forestales em contenedor. Madrid: Ministério de Agricultura, Pesca y Alimentación. Secretaria General Técnica Centro de Publicaciones, 1996. 189p.

Sanford, R. L.; Cuevas, E. Root growth and rhizosphere interactions in tropical forests. In: Mulkey, S. S.; Chazdon, R. L.; Smith, A. P. (eds.). Tropical forest plant ecophysiology. Chapman \& Hall, New York, 1996. p. 268-300.

Scalon, S. P. Q.; Mussury, R. M.; Euzébio, V. L. M.; Kodama, F. M.; KISSMANN, C. Estresse hídrico no metabolismo e crescimento inicial de mudas de mutambo (Guazuma ulmifolia Lam.). Ciência Florestal, v.21, n.4, p.655-662, 2011. https://periodicos.ufsm.br/cienciaflorestal/article/view/45 $\underline{10 / 2671}$

Silva, F. A. S.; Azevedo,C. A. V. Versão do programa computacional Assistat para o sistema operacional Windows. Revista Brasileira de Produtos Agroindustriais, v.4, n.1, p.71-78, 2002.

http://www.deag.ufcg.edu.br/rbpa/rev41/Art410.pdf

Silva, G. H.; Santos, R. V.; Gomes, A. D. V. Crescimento de mudas de craibeira em substrato de co-produto sob fertilização química e orgânica. Revista Verde de Agroecologia e Desenvolvimento Sustentável, v.9, n.5, p.7883 ,

2014.
https://www.gvaa.com.br/revista/index.php/RVADS/artic le/view/2599/3040

Silva, M. R.; Klar, A. E.; Passos, J. R. Efeitos do manejo hídrico e da aplicação de potássio nas características morfofisiológicas de mudas de Eucalyptus grandis W. (Hill ex. Maiden). Irriga, v.9, n.1, p.31-40, 2004. https://doi.org/10.15809/irriga.2004v9n1p31-40

Simões, D.; Silva, R. B. G.; Silva, M. R. Composição do substrato sobre o desenvolvimento, qualidade e custo de produção de mudas de Eucalyptus grandis Hill ex Maiden $\times$ Eucalyptus urophylla S. T. Blake. Ciência Florestal, v.22, n.1, p.91-100, 2012. http://dx.doi.org/10.5902/198050985082

Souza, C. C.; Oliveira, F. A.; Silva, I. F.; Amorim Neto, M. S. Avaliação de métodos de determinação de água disponível e manejo da irrigação em terra roxa sob cultivo de algodoeiro herbáceo. Revista Brasileira de Engenharia Agrícola e Ambiental, v.4, n.3, p.338-342,

2000 .

http://www.scielo.br/pdf/\%0D/rbeaa/v4n3/v4n3a06.pdf

Taiz, L.; Zeiger, E. Fisiologia vegetal. 5. ed. Porto Alegre: Artmed, 2013. 918 p.

Toledo, F. H.; Venturin, N.; Carlos, L.; Dias, B. A.; Venturin, R. P.; Macedo, R. L. Compost of residues of pulp and paper in the production of eucalyptus seedlings. Revista Brasileira de Engenharia Agrícola e Ambiental, v.19, n.7, p.711-716, 2015. http://dx.doi.org/10.1590/18071929/agriambi.v19n7p711-716

Trajano, E. V. A.; Santos, R. V.; Bakke, O. A.; Vital, A. F. M.; Santos, Y. M.; Quaresma, J. M.; Salviano, V. M. Crescimento do pinhão manso em substratos com rejeitos de mineração do semi-árido-PB. In: CONGRESSO BRASILEIRO DE MAMONA, 4 \& SIMPÓSIO INTERNACIONAL DE OLEAGINOSAS ENERGÉTICAS, 1., 2010, João Pessoa. Anais... João Pessoa: Empraba Algodão, 2010. p. 545-550. https://www.alice.cnptia.embrapa.br/bitstream/doc/85552 5/1/FER09.pdf

Villagra, P. E.; Cavagnaro, J. B. Water stress effects on the seedling growth of Prosopis argentina and Prosopis alpataco. Journal of Arid Environments, v.64, n.3, p.390-400, 2006. https://doi.org/10.1016/j.jaridenv.2005.06.008 